\title{
Blow-up profile to solutions of NLS with oscillating nonlinearities
}

\author{
Jian Zhang and Shihui Zhu
}

\begin{abstract}
This paper is concerned with the blow-up solutions of nonlinear Schrödinger equation (NLS) with oscillating nonlinearities. The limiting profiles of the blow-up solutions $u(t, x)$ with initial data $\left\|u_{0}\right\|_{L^{2}}=\|Q\|_{L^{2}}$ are obtained. It reads that $|u(t, x)|^{2} \rightarrow\|Q\|_{L^{2}}^{2} \delta_{x=y_{1}}$ (Dirac function), as $t \rightarrow T$, and that $u(t, x)$ converges strongly to $Q(x)$ in the energy space $\Sigma=\left\{u \in H^{1} ; \int|x|^{2}|u|^{2} d x<\infty\right\}$ up to scaling and phase parameters and also translation in the nonradial case.
\end{abstract}

Mathematics Subject Classification (2010). 35Q55, 35B35.

Keywords. Nonlinear Schrödinger equation, Oscillating nonlinearity, Blow-up solution, Blow-up profile.

\section{Introduction}

In this paper, we study the Cauchy problem of the following critical nonlinear Schrödinger equation with multiplicative oscillating term

$$
\begin{gathered}
i u_{t}+\Delta u+\cos ^{2}(\omega t)|u|^{\frac{4}{N}} u=0, \quad t \in\left[t_{0}, T\right), x \in R^{N}, \\
u\left(t_{0}, x\right)=u_{0},
\end{gathered}
$$

where $\omega$ is a positive parameter; $i=\sqrt{-1} ; \triangle=\sum_{j=1}^{N} \frac{\partial^{2}}{\partial x_{j}^{2}}$ is the Laplace operator in $R^{N} ; u=u(t, x):\left[t_{0}, T\right) \times R^{N} \rightarrow C$ is the complex valued function and $t_{0}<T \leq \infty ; N$ is the space dimension. Equation (1.1) is also called nonlinear Schrödinger equation with time dependent potential, which may model a laser beam that propagates in a medium with variable indices of refraction and has received a lot of attentions recently (see [10-12,20,26]). In Physics, the blow-up of solutions corresponds to the wave's collapse, physicists are very interested in the elaborate descriptions of the blow-up solutions.

Supported by National Science Foundation of P. R. China (11071177). 
We recall the classical nonlinear Schrödinger equation without any potential. Ginibre and Velo [5] showed the local well-posedness in $H^{1}\left(R^{N}\right)$. Glassey [6], Ogawa and Tsutsumi [18] showed the existence of blow-up solutions (see Cazenave [2] for a review). Weinstein [21] and Zhang [23] obtained the sharp conditions of the blow-up and global existence. Weinstein [22] showed the structure and formation of singularity to the blow-up solution with critical mass $\left(\|Q\|_{L^{2}}\right)$, and it reads that the blow-up solution is close to $Q(x)$ in $H^{1}\left(R^{N}\right)$ up to scaling and phase parameters, and also translation in the nonradial case. Based on this property, Merle and Tsutsumi [13] showed a new phenomenon for the blow-up solutions which is the $L^{2}$-concentration. Merle [14] obtained the exact blow-up solution with critical mass by the conformal invariance and compact results. Recently, using the variational methods, Merle and Raphaël [15] improved the Weinstein's [22] results and obtained the sharp decomposition of blow-up solutions with small super-critical mass. Thus, basing on this sharp decomposition, Merle and Raphaël [15-17] obtained a large body of breakthrough work with the help of the Spectral Properties, such as blow-up rates, profiles of blow-up solutions, etc. Hmidi and Keraani [8] used a profile decomposition of bounded sequences in $H^{1}\left(R^{N}\right)$ proposed by Gérard [7] to obtain a refined version of compactness lemma, and gave a direct proof of the results obtained by Weinstein [22].

For Eq. (1.1), Cazenave [2] established the local well-posedness in the corresponding energy field. Few results about the blow-up solutions of the Cauchy problem (1.1)-(1.2) are known. Damergi and Goubet [4] showed the existence of blow-up solutions for some initial data. Sulem [20] obtained some numerical observations. It is obvious that conservation laws are vital in studying the blow-up solution of the nonlinear Schrodinger equation, but there is no conservation of energy for the Eq. (1.1) because of the effects of the oscillating Nonlinearities. Therefore, How to deal with the loss of conservation of energy for the Cauchy problem (1.1)-(1.2) is of particular interest. Motivated by these problems, we seek to answer the following question: what is the limiting profile of the blow-up solutions for the Cauchy problem (1.1)-(1.2)?

In this paper, in terms of Zhang's [24] ideas, we choose the ground state solution of the nonlinear Schrödinger equation without any potential as the limiting profiles to describe the blow-up solutions of Cauchy problem (1.1)-(1.2). Thus, using the compactness lemma proposed by Hmidi and Keraani [8] and the variational characterization of the corresponding ground state solution, we obtain the shape of the blow-up solutions for the initial data $\left\|u_{0}\right\|_{L^{2}} \geq\|Q\|_{L^{2}}$. Specifically, suppose that the initial data $u_{0} \in \Sigma,\left\|u_{0}\right\|_{L^{2}}=$ $\|Q\|_{L^{2}}$, and the corresponding solution $u(t, x)$ blows up in finite time $T<\infty$. Then there exists an $y_{1} \in R^{N}$ such that

$$
|u(t, x)|^{2} \rightarrow\|Q(x)\|_{L^{2}}^{2} \delta_{x=y_{1}}, \text { as } \mathrm{t} \rightarrow \mathrm{T} .
$$

Applying these properties, we obtain the limiting profiles of blow-up solutions in the energy space $\Sigma$. There are functions $y(t) \in R^{N}, \gamma(t) \in R$ such that

$$
\lambda^{\frac{N}{2}}(t) u(t, \lambda(t)(x+y(t))) e^{i \gamma(t)} \rightarrow Q(x) \text { strongly in } \Sigma \text { as } t \rightarrow T,
$$


where $\lambda(t)=\frac{\|\nabla Q\|_{L^{2}}}{\|\nabla u\|_{L^{2}}}$ and $Q(x)$ is the unique ground state solution of the following equation (see [9])

$$
-\triangle Q+Q-|Q|^{\frac{4}{N}} Q=0, \quad Q \in H^{1}\left(R^{N}\right) .
$$

Moreover, we extend the results in (1.4) to the case: $\|Q\|_{L^{2}}<\left\|u_{0}\right\|_{L^{2}}<$ $\|Q\|_{L^{2}}+\alpha^{\prime}$.

The aim of this paper is to obtain the blow-up profile to the blow-up solutions of the Cauchy problem (1.1)-(1.2) in the energy space $\Sigma:=\{u \in$ $\left.H^{1}\left(R^{N}\right),|x| u \in L^{2}\left(R^{N}\right)\right\}$. Zhu, Zhang and Li [25] obtained the limiting profile of the blow-up solutions for the nonlinear Schrödinger equation with a harmonic potential (space dependent potential). Chen and Guo [3] obtained the blow-up profile to the solutions of the coupled Schrödinger equations. The main idea of this paper is from our previous paper [25], but there are two marked differences between this paper and the previous paper [25]. One is that the equation dealt with in this paper is different from that in the paper [25]. In the paper [25], authors studied the Gross-Potaevskii equation, which is a class of nonlinear Schrödinger equations with a space dependent potential and there is the conservation of energy. In this paper, we consider the NLS with oscillating nonlinearity, which is a class of nonlinear Schrödinger equation with a time dependent nonlinearity. Because of the time dependent nonlinearity, there is no conservation of energy for the NLS with oscillating nonlinearity, which is one of the main technique challenges in this paper. The other is that the tool used in this paper is different from that in the paper [25]. In the paper [25], authors used the classical concentration compactness principle proposed by Lions. In this paper, we apply the refined compactness lemma proposed by Hmidi and Keraani [8] to study the limiting profile and obtain that the blowup solution is like a Dirac function (see (1.3)). Furthermore, it is easy to check $\int|x|^{2}|u(t, x)|^{2} d x$ is bounded, but after scaling $U(t, x)=\lambda^{\frac{N}{2}}(t) u(t, \lambda(t) x)$ it is difficult to control $U(t, x)$ in the weighed Sobolev space $\Sigma$, and we need a new estimate to obtain the limiting profile of the blow-up solutions in $\Sigma$.

In this paper, we denote $L^{q}\left(R^{N}\right),\|\cdot\|_{L^{q}\left(R^{N}\right)}, H^{s}\left(R^{N}\right)$ and $\int_{R^{N}} \cdot d x$ by $L^{q},\|\cdot\|_{L^{q}}, H^{s}$ and $\int \cdot d x$ respectively, and the various positive constants will be simply denoted by $C$.

\section{Preparation}

For the Cauchy problem (1.1)-(1.2), we define the energy space $\Sigma:=\{u \in$ $\left.H^{1}\left(R^{N}\right)|| x \mid u \in L^{2}\left(R^{N}\right)\right\}$ with the inner product

$$
\langle u, v\rangle=\int\left(\nabla u \nabla \bar{v}+u \bar{v}+|x|^{2} u \bar{v}\right) d x .
$$

The norm of $\Sigma$ is denoted by $\|\cdot\|_{\Sigma}$. Moreover, we define two functionals on $\Sigma$ by

$$
\begin{aligned}
& E(u):=\int|\nabla u|^{2} d x-\frac{\cos ^{2}(\omega t)}{1+\frac{2}{N}} \int|u|^{2+\frac{4}{N}} d x, \\
& H(u):=\int|\nabla u|^{2} d x-\frac{1}{1+\frac{2}{N}} \int|u|^{2+\frac{4}{N}} d x .
\end{aligned}
$$


The functionals $E$ and $H$ are well-defined according to the Sobolev embedding theorem (see [2]). Cazenave [2] showed the local well-posedness for the Cauchy problem (1.1)-(1.2) in $\Sigma$, as follows.

Proposition 2.1. (Cazenave [2]) Let $u_{0} \in \Sigma$. There exists an unique solution $u(t, x)$ of the Cauchy problem (1.1)-(1.2) on the maximal time $\left[t_{0}, T\right)$ such that $u(t, x) \in C\left(\left[t_{0}, T\right) ; \Sigma\right)$ and either $T=\infty$ (global existence), or else $T<\infty$ and $\lim _{t \rightarrow T}\|u(t, x)\|_{\Sigma}=\infty$ (wave's collapse). Furthermore, for all $t \in\left[t_{0}, T\right), u(t, x)$ satisfies the following conservation law

$$
\|u(t, x)\|_{L^{2}}=\left\|u_{0}\right\|_{L^{2}} .
$$

From now on, we give some important lemmas.

Lemma 2.1. (Strauss [19] Kwong [9]) There exists an unique ground state solution $Q(x)$ to Eq. (1.5) which is positive and spherically symmetric and exponentially decays at infinity.

Lemma 2.2. (Weinstein [21]) Let $u \in H^{1}$, then

$$
H(u) \geq\left[1-\left(\frac{\|u\|_{L^{2}}}{\|Q\|_{L^{2}}}\right)^{\frac{4}{N}}\right]\|\nabla u\|_{L^{2}}^{2},
$$

where $Q$ is the unique ground state solution of Eq. (1.5).

Lemma 2.3. (Hmidi and Keraani [8]) Let $\left\{v_{n}\right\}$ be a bounded family of $H^{1}$ such that

$$
\lim _{n \rightarrow \infty} \sup \left\|\nabla v_{n}\right\|_{L^{2}} \leq M<\infty
$$

and

$$
\lim _{n \rightarrow \infty} \sup \left\|v_{n}\right\|_{L^{2+\frac{4}{N}}} \geq m>0 .
$$

Then there exists $\left\{x_{n}\right\} \subset R^{N}$ such that, up to a subsequence,

$$
v_{n}\left(x+x_{n}\right) \rightarrow V(x), \quad \text { weakly in } H^{1}
$$

with $\|V\|_{L^{2}} \geq\left(\frac{2 N}{N+2}\right)^{\frac{N}{4}} \frac{m^{1+N / 2}}{M^{N / 2}}\|Q\|_{L^{2}}$.

Lemma 2.4. Let $u_{0} \in \Sigma$, and $u(t, x) \in C\left(\left[t_{0}, T\right) ; \Sigma\right)$ be the solution of the Cauchy problem (1.1)-(1.2). Then, $\forall t \in\left[t_{0}, T\right)$, one has

$$
\frac{d^{2}}{d t^{2}} \int|x|^{2}|u(t, x)|^{2} d x=16 E(u(t, x)) .
$$

Moreover, there exists an $y_{1} \in R^{N}$ such that

$$
\lim _{t \rightarrow T} \int|u(t, x)|^{2} x d x=-\left(\int|Q(x)|^{2} d x\right) y_{1},
$$

where $Q(x)$ is the unique ground state solution of Eq. (1.5). 
Proof. Since $u_{0} \in \Sigma$ and $u(t, x) \in C\left(\left[t_{0}, T\right) ; \Sigma\right)$ is the solution of the Cauchy problem (1.1)-(1.2), $J(t):=\int|x|^{2}|u(t, x)|^{2} d x$ is well-defined. By some computations, one has for all $t \in\left[t_{0}, T\right)$

$$
\begin{aligned}
J^{\prime}(t) & =2 \Re \int|x|^{2} \bar{u} u_{t} d x \\
& =-2 \Im \int|x|^{2} \bar{u}\left(\triangle u+\cos ^{2}(\omega t)|u|^{\frac{4}{N}} u\right) d x \\
& =4 \Im \int x \cdot \nabla u \bar{u} d x,
\end{aligned}
$$

and

$$
J^{\prime \prime}(t)=-4 N \Im \int \bar{u} u_{t} d x-8 \Im \int x \nabla \bar{u} u_{t} d x .
$$

Since $u(t, x)$ is the solution of (1.1), one deduces that

$$
\Im \int \bar{u} u_{t} d x=-\int|\nabla u|^{2} d x+\cos ^{2}(\omega t) \int|u|^{2+\frac{4}{N}} d x,
$$

and

$$
\Im \int x \nabla \bar{u} u_{t} d x=\frac{N-2}{2} \int|\nabla u|^{2} d x-\frac{N}{2+\frac{4}{N}} \cos ^{2}(\omega t) \int|u|^{2+\frac{4}{N}} d x .
$$

By (2.9)-(2.11) and the definition of the energy functional $E(u)$, one has

$$
J^{\prime \prime}(t)=16 E(u),
$$

which implies (2.6) is true.

On the other hand, for any $x_{0} \in R^{N}$, let $g(t):=\int x_{0} \cdot x|u(t, x)|^{2} d x$, then

$$
g^{\prime}(t)=2 \Im \int x_{0} \cdot \nabla u \bar{u} d x
$$

thus, one gets

$$
\begin{aligned}
g^{\prime \prime}(t) & =-4 \Im \int x_{0} \nabla \bar{u} u_{t} d x \\
& =-4 \Im \int x_{0} \nabla \bar{u} i\left(\triangle u+\cos ^{2}(\omega t)|u|^{\frac{4}{N}} u\right)=0 .
\end{aligned}
$$

Hence, one has

$$
g(t)=x_{0} \int x\left|u_{0}\right|^{2} d x+\left(t-t_{0}\right) x_{0} \Im \int x \nabla u_{0} \overline{u_{0}} d x .
$$

Since $x_{0} \in R^{N}$ is arbitrary, one deduces that $\forall t \in\left[t_{0}, T\right]$

$$
\int x|u(t, x)|^{2} d x=\int x\left|u_{0}\right|^{2} d x+2\left(t-t_{0}\right) \Im \int x \nabla u_{0} \overline{u_{0}} d x .
$$

Therefore, one can choose a proper $y_{1} \in R^{N}$ such that

$$
\lim _{t \rightarrow T} \int x|u(t, x)|^{2} d x=-\left(\int|Q(x)|^{2} d x\right) y_{1} .
$$

This completes the proof. 


\section{Concentration property of blow-up solution}

In this section, we further study the concentration properties of blow-up solutions with initial data $\left\|u_{0}\right\|_{L^{2}}=\|Q\|_{L^{2}}$. Using the variational characterization of the corresponding ground state, we obtain that if the initial data $u_{0} \in \Sigma$ with $\left\|u_{0}\right\|_{L^{2}}=\|Q\|_{L^{2}}$, then the blow-up solution remains close to $Q$ in $H^{1}\left(R^{N}\right)$ up to scaling and phase parameters, and also translation in the nonradial case. Moreover, using these properties of blow-up solutions, we obtain that the blow-up solution $|u(t, x)|^{2}$ like a $\delta$-function as $t \rightarrow T$ at the point $x=y_{1}$, which implies that the point $y_{1}$ concentrates all mass of blow-up solution of the Cauchy problem (1.1)-(1.2).

Proposition 3.1. Let $u_{0} \in \Sigma$ and $\left\|u_{0}\right\|_{L^{2}}=\|Q\|_{L^{2}}$. Suppose that $t_{0}=\frac{\pi}{2 \omega}$ and the corresponding solution of the Cauchy problem (1.1)-(1.2) $u(t, x)$ blows up in finite time $T \leq \frac{\pi}{\omega}$. Then there are functions $y(t) \in R^{N}, \gamma(t) \in R$ such that

$\lambda^{\frac{N}{2}}(t) u(t, \lambda(t)(x+y(t))) e^{i \gamma(t)} \rightarrow Q(x)$, strongly in $H^{1} \quad$ as $\quad t \rightarrow T$,

where $\lambda(t)=\frac{\|\nabla Q\|_{L^{2}}}{\|\nabla u\|_{L^{2}}}$ and $Q(x)$ is the unique ground state solution of $E q$. (1.5).

Proof. We prove Proposition 3.1 by contradiction. We show that for any sequence $t_{k} \rightarrow T$, there are $y_{k}$ and $\gamma\left(t_{k}\right)$ such that

$$
\lambda^{\frac{N}{2}}\left(t_{k}\right) v\left(t_{k}, \lambda\left(t_{k}\right)\left(x+y\left(t_{k}\right)\right)\right) e^{i \gamma\left(t_{k}\right)} \rightarrow Q(x) \quad \text { strongly in } H^{1} \text { as } k \rightarrow \infty,
$$

where $Q(x)$ is the ground state solution of Eq. (1.5). If not, then (3.2) does not holds along some sequence $t_{k}$. But then we can find a subsequence of $t_{k}$ along with (3.2) holds, this is a contradiction. Since $t_{k}$ is an arbitrary sequence approaching $T$, and (3.1) follows.

Since $u(t, x)$ is the solution of the Eq. (1.1), multiplying (1.1) with $u_{t}$ and integrating, one has that

$$
\frac{d}{d t} \int|\nabla u|^{2} d x-\frac{\cos ^{2}(\omega t)}{1+\frac{2}{N}} \frac{d}{d t} \int|u|^{2+\frac{4}{N}} d x=0
$$

which implies that

$$
\frac{d}{d t} E(u(t))=\frac{\omega}{1+\frac{2}{N}} \sin (2 \omega t) \int|u|^{2+\frac{4}{N}} d x .
$$

By $\frac{\pi}{2 \omega}=t_{0} \leq t<T \leq \frac{\pi}{\omega}$, one has that $\frac{d}{d t} E(u(t)) \leq 0$ and

$$
E(u(t)) \leq E\left(u_{0}\right), \text { for } t_{0}<t<T .
$$

Since the solution $u(t, x) \in C\left(\left[t_{0}, T\right) ; \Sigma\right)$ blows up in finite time $T<\frac{\pi}{\omega}$, satisfying $\lim _{t \rightarrow T}\|\nabla u\|_{L^{2}}=\infty$. For any $t_{k} \rightarrow T$ as $k \rightarrow \infty$, one takes

$$
\frac{1}{\lambda_{k}^{2}}=\int\left|\nabla u\left(x, t_{k}\right)\right|^{2} d x \rightarrow \infty, \text { as } \mathrm{k} \rightarrow \infty .
$$


Considering $U_{k}=\lambda_{k}^{\frac{N}{2}} u\left(\lambda_{k} x\right)$, by direct computations, one has

$$
\left\{\begin{array}{l}
\left\|U_{k}\right\|_{L^{2}}=\left\|u\left(t_{k}\right)\right\|_{L^{2}}=\left\|u_{0}\right\|_{L^{2}}=\|Q(x)\|_{L^{2}}, \\
\left\|\nabla U_{k}\right\|_{L^{2}}=\lambda_{k}\left\|\nabla u\left(t_{k}\right)\right\|_{L^{2}}=1 .
\end{array}\right.
$$

Therefore, $U_{k}$ is a uniformly bounded sequence in $H^{1}$. Note that

$$
\begin{aligned}
H\left(U_{k}\right) & =\int\left|\nabla U_{k}\right|^{2} d x-\frac{1}{1+\frac{2}{N}} \int\left|U_{k}\right|^{2+\frac{4}{N}} d x \\
& =\lambda_{k}^{2} \int\left|\nabla u\left(t_{k}\right)\right|^{2}-\frac{1}{1+\frac{2}{N}} \lambda_{k}^{2} \int\left|u\left(t_{k}\right)\right|^{2+\frac{4}{N}} d x \\
& \leq \lambda_{k}^{2} \int\left|\nabla u\left(t_{k}\right)\right|^{2}-\frac{\cos ^{2}\left(\omega t_{k}\right)}{1+\frac{2}{N}} \lambda_{k}^{2} \int\left|u\left(t_{k}\right)\right|^{2+\frac{4}{N}} d x \\
& \leq \lambda_{k}^{2} E\left(u_{0}\right) \rightarrow 0, \text { as } k \rightarrow \infty .
\end{aligned}
$$

On the other hand, by (3.6) and Gagliardo-Nirenberg inequality, one has that $H\left(U_{k}\right) \geq 0$ and

$$
\lim _{k \rightarrow \infty} H\left(U_{k}\right)=0
$$

Combining (3.8) with (3.7), one has

$$
\lim _{k \rightarrow \infty}\left\|U_{k}\right\|_{L^{2+\frac{4}{N}}}^{2+\frac{4}{N}}=1+\frac{2}{N} .
$$

Applying Lemma 2.4 to the sequence $U_{k}\left(M=1, m^{2+4 / N}=1+\frac{2}{N}\right)$, one has that there exist $\left\{y_{k}\right\} \subset R^{N}$ and $U \in H^{1}$ such that

$$
U_{k}\left(x+y_{k}\right) \rightarrow U(x) \text { weakly in } H^{1}
$$

with $\|U\|_{L^{2}} \geq\|Q\|_{L^{2}}$. Since $\|U\|_{L^{2}} \leq\left\|U_{k}\left(x+y_{k}\right)\right\|_{L^{2}}=\|Q\|_{L^{2}}$ and the BrézisLieb Lemma (see [1]), one has

$$
U_{k}\left(x+y_{k}\right) \rightarrow U(x) \text { strongly in } L^{2} .
$$

By the Gagliardo-Nirenberg's inequality (see Lemma 2.3), there exists $\gamma_{k} \in R$ such that

$\left\|U_{k}\left(x+y_{k}\right) e^{i \gamma_{k}}-U\right\|_{L^{2+\frac{4}{N}}}^{2+\frac{4}{N}} \leq C\left\|U_{k}\left(x+y_{k}\right) e^{i \gamma_{k}}-U\right\|_{L^{2}}^{\frac{4}{N}}\left\|\nabla\left(U_{k}\left(x+y_{k}\right) e^{i \gamma_{k}}-U\right)\right\|_{L^{2}}^{2}$. It follows from $(3.9)$ and $\left\|\nabla U_{k}\left(x+y_{k}\right)\right\|_{L^{2}} \leq C$ that

$$
U_{k}\left(x+y_{k}\right) e^{i \gamma_{k}} \rightarrow U \text { strongly in } L^{2+\frac{4}{N}} .
$$

Next, we shall show that $U_{k}\left(x+y_{k}\right) e^{i \gamma_{k}}$ converges to $U$ strongly in $H^{1}$. We need only now show that $\|\nabla U\|_{L^{2}}=1$ by the Brézis-Lieb Lemma (see [1]). Note that

$$
\begin{aligned}
0 & =\lim _{k \rightarrow \infty} H\left(U_{k} e^{i \gamma_{k}}\right) \\
& =1-\frac{1}{1+\frac{2}{N}} \lim _{k \rightarrow \infty}\left\|U_{k}\right\|_{L^{2+\frac{4}{N}}}^{2+\frac{4}{N}} \\
& =1-\frac{1}{1+\frac{2}{N}}\|U\|_{L^{2+\frac{4}{N}}}^{2+\frac{4}{N}}
\end{aligned}
$$


Hence, if $\|\nabla U\|_{L^{2}}<1$, by (3.11) one has $H(U)<0$, this is impossible by Lemma 2.3 and the fact $U \not \equiv 0$. On the other hand, if $\|\nabla U\|_{L^{2}}>1$, by (3.11) one has $H(U)>0$. Therefore, we have showed that $\|\nabla U\|_{L^{2}}=1$, which implies that $U_{k}\left(x+y_{k}\right) e^{i \gamma k}$ converges strongly to $U$ in $H^{1}$.

Applying $\left\|u_{0}\right\|_{L^{2}}=\|Q\|_{L^{2}}$, (3.6)and (3.7), one deduces that $U_{k_{j}}$ is a minimizing sequence of the variational problem

$$
I\left(\|Q\|_{L^{2}}\right) \equiv \inf \left\{H(f) \mid f \in H^{1},\|f\|_{L^{2}}=\|Q\|_{L^{2}}\right\} .
$$

Moreover, one obtains that $U$ is a minimizer of variational problem (3.12) and $|U|$ is also a minimizer of (3.12). Indeed, since $\|\nabla|U|\|_{L^{2}} \leq\|\nabla U\|_{L^{2}}$, one gets

$$
H(|U|) \leq H(U)=0 .
$$

It is obvious that $\|U\|_{L^{2}}=\|\mid U\|_{L^{2}}=\|Q\|_{L^{2}}$, and one has $H(|U|) \geq 0$ by Lemma 2.3. Hence, $H(|U|)=0$.

Therefore, applying the variational characteristic of the ground state of Eq. (1.5), one deduces that, $\exists y \in R^{N}, \gamma \in R$ such that $U(x)=Q(x+y) e^{i \gamma}$, which implies that

$\lambda_{k}^{\frac{N}{2}} u\left(t_{k}, \lambda_{k}\left(x+y_{k}\right)\right) e^{i \gamma_{k}} \rightarrow Q(x+y) e^{i \gamma}$ strongly in $H^{1}$ as $k \rightarrow \infty$.

By redefining the sequences $y_{k}$ and $\gamma_{k}$, we have (3.2) is true. This completes the proof of Proposition 3.1.

In the end, we using the Proposition 3.1 to obtain the following concentration properties of the blow-up solutions for the Cauchy problem (1.1)-(1.2): The blow-up solution converges to a Dirac function when $t$ closes to the blowup time $T$.

Theorem 3.2. Let $u_{0} \in \Sigma$ with $\left\|u_{0}\right\|_{L^{2}}=\|Q\|_{L^{2}}$. Suppose that $t_{0}=\frac{\pi}{2 \omega}$ and the corresponding solution of the Cauchy problem (1.1)-(1.2) u(t,x) blows up in finite time $T \leq \frac{\pi}{\omega}$. Then one has

$$
|u(t, x)|^{2} \rightarrow\|Q(x)\|_{L^{2}}^{2} \delta_{x=y_{1}}, \text { as } \mathrm{t} \rightarrow \mathrm{T} .
$$

where $\lambda(t)=\frac{\|\nabla Q\|_{L^{2}}}{\|\nabla u\|_{L^{2}}}, Q(x)$ is the unique ground state solution of $E q$. (1.5) and $y_{1} \in R^{N}$ is defined in Lemma 2.5.

Proof. Applying Proposition 3.1, one has that there exist functions $\gamma(t) \in R$, $y(t) \in R^{N}$ such that

$$
e^{i \gamma(t)} \lambda^{\frac{N}{2}}(t) u(t, \lambda(t)(x+y(t))) \rightarrow Q(x) \text { in } H^{1} \quad \text { as } t \rightarrow T,
$$

where $\lambda(t)=\frac{\|\nabla Q\|_{L^{2}}}{\|\nabla u\|_{L^{2}}} \rightarrow 0$, as $t \rightarrow T$.

Therefore,

$$
\lambda^{N}(t)|u(t, \lambda(t)(x+y(t)))|^{2} \rightarrow|Q(x)|^{2} \text { in } \mathrm{L}^{1}, \text { as } \mathrm{t} \rightarrow \mathrm{T},
$$

and

$$
|u(t, x+y(t))|^{2} \rightarrow\|Q(x)\|_{L^{2}}^{2} \delta_{x=0}, \quad \text { as } \mathrm{t} \rightarrow \mathrm{T},
$$

by the fact $\|\nabla u(t, x)\|_{L^{2}} \rightarrow \infty$, as $t \rightarrow T$. 
Applying (2.6) and (3.5), one has that

$$
\frac{d^{2}}{d t^{2}} \int|x|^{2}|u(t, x)|^{2} d x \leq 16 E\left(u_{0}\right)
$$

which implies that there exists a constant $C_{2}>0$ such that

$$
\forall t \in\left[t_{0}, T\right), \quad \int|x|^{2}|u(t, x)|^{2} d x \leq C_{2} .
$$

Hence, there exists a $C>0$ such that

$$
C \int|y(t)|^{2}|u(t, x)|^{2} d x \leq \int|x+y(t)|^{2}|u(t, x+y(t))|^{2} d x \leq C_{2} .
$$

Hence, one deduces that

$$
\varlimsup_{t \rightarrow T}|y(t)| \leq \frac{\sqrt{C_{2} / C}}{\|Q(x)\|_{L^{2}}},
$$

and there exists an $R_{0}>0, \forall t \in\left[t_{0}, T\right)$

$$
|y(t)| \leq R_{0}
$$

We claim that for $\forall R>R_{0}$

$$
\lim _{t \rightarrow T}\left\{\int_{B(0, R)}|u(t, x)|^{2} x d x-\left(\int|Q(x)|^{2} d x\right) y(t)\right\}=0,
$$

where $B(0, R)=\left\{x \in R^{N}|| x \mid \leq R\right\}$ is the ball of radius $R$ centered at the origin. Indeed,

$$
\begin{aligned}
\int_{B(0, R)}|u(t, x)|^{2} x d x= & \int_{B(0, R)}|u(t, x)|^{2}(x-y(t)) d x+\int_{B(0, R)}|u(t, x)|^{2} y(t) d x \\
= & \int_{B(-y(t), R)}|u(t, y+y(t))|^{2} y d y \\
& +\int_{B(-y(t), R)}|u(t, y+y(t))|^{2} y(t) d y \\
= & \int_{B(0, \delta)}|u(t, y+y(t))|^{2} y d y \\
& +\int_{B(-y(t), R) \backslash B(0, \delta)}|u(t, y+y(t))|^{2} y d y \\
& +\int_{B(-y(t), R)}|u(t, y+y(t))|^{2} y(t) d y .
\end{aligned}
$$

Because of $R>R_{0}$, there exists a $\delta>0, B(0, \delta) \subset B(-y(t), R)$, by $(3.15)$, one has

$$
\int_{B(0, R)}|u(t, x)|^{2} x d x-\|Q(x)\|_{L^{2}}^{2} y(t) \rightarrow 0, \text { as } \mathrm{t} \rightarrow \mathrm{T},
$$

this completes the proof of Claim (3.19). 
On the other hand, by (3.16), one has $\forall t \in\left[t_{0}, T\right)$

$$
\left.\left|\int_{|x| \geq R}\right| u(t, x)\right|^{2} x d x \mid \leq \frac{C_{2}}{R^{2}} .
$$

Thus, (3.20) and (3.21) implies that

$$
\lim _{t \rightarrow T}\left\{\int|u(t, x)|^{2} x d x-\left(\int|Q(x)|^{2} d x\right) y(t)\right\}=0 .
$$

Since Lemma 2.5, there exists a $y_{1} \in R^{N}$ such that

$$
\lim _{t \rightarrow T} \int|u(t, x)|^{2} x d x=-\left(\int|Q(x)|^{2} d x\right) y_{1} .
$$

It follows from (3.22)-(3.23) that

$$
y(t) \rightarrow y_{1}, \quad \text { as } \mathrm{t} \rightarrow \mathrm{T},
$$

and

$$
\left|u\left(t, x-y_{1}\right)\right|^{2} \rightarrow\|Q(x)\|_{L^{2}}^{2} \delta_{x=0}, \text { as } \mathrm{t} \rightarrow \mathrm{T} .
$$

Therefore, one obtains

$$
|u(t, x)|^{2} \rightarrow\|Q(x)\|_{L^{2}}^{2} \delta_{x=y_{1}}, \text { as } \mathrm{t} \rightarrow \mathrm{T},
$$

which concludes the proof of Theorem 3.2.

At the end of this paper, we show the $L^{2}$-Concentration properties of blow-up solution by the compactness lemma proposed by Hmidi and Keraani [8].

Theorem 3.3. Let $u_{0} \in \Sigma$ and $t_{0}=\frac{\pi}{2 \omega}$. Assume the corresponding solution of the Cauchy problem (1.1)-(1.2) $u(t, x)$ blows up in finite time $T \leq \frac{\pi}{\omega}$. Suppose that $a(t)>0$ is any function such that $a(t)\|\nabla u(t)\|_{L^{2}} \rightarrow \infty$ as $t \rightarrow T$. Then, there exists $y(t) \in R^{N}$ such that

$$
\lim \inf _{t \rightarrow T} \int_{|x-y(t)| \leq a(t)}|u(t, x)|^{2} d x \geq \int Q^{2} d x,
$$

where $Q(x)$ is the unique ground state solution of Eq. (1.5).

Proof. Since $u(t, x) \in C\left(\left[t_{0}, T\right) ; \Sigma\right)$ blows up in finite time $T<\frac{\pi}{\omega}$ satisfying $\lim _{t \rightarrow T}\|\nabla u\|_{L^{2}}=\infty$. For any $t_{k} \rightarrow T$ as $k \rightarrow \infty$, we take

$$
\frac{1}{\lambda_{k}^{2}}=\int\left|\nabla u\left(x, t_{k}\right)\right|^{2} d x \rightarrow \infty, \text { as } \mathrm{k} \rightarrow \infty
$$

Considering $U_{k}=\lambda_{k}^{\frac{N}{2}} u\left(\lambda_{k} x\right)$, by direct computations, one has

$$
\left\{\begin{array}{l}
\left\|U_{k}\right\|_{L^{2}}=\left\|u\left(t_{k}\right)\right\|_{L^{2}}=\left\|u_{0}\right\|_{L^{2}}=\|Q(x)\|_{L^{2}}, \\
\left\|\nabla U_{k}\right\|_{L^{2}}=\lambda_{k}\left\|\nabla u\left(t_{k}\right)\right\|_{L^{2}}=1 .
\end{array}\right.
$$


Therefore, $U_{k}$ is a uniformly bounded sequence in $H^{1}$. It follows from (3.5) that

$$
\begin{aligned}
H\left(U_{k}\right) & =\int\left|\nabla U_{k}\right|^{2} d x-\frac{1}{1+\frac{2}{N}} \int\left|U_{k}\right|^{2+\frac{4}{N}} d x \\
& \leq \lambda_{k}^{2} \int\left|\nabla u\left(t_{k}\right)\right|^{2}-\frac{\cos ^{2}\left(\omega t_{k}\right)}{1+\frac{2}{N}} \lambda_{k}^{2} \int\left|u\left(t_{k}\right)\right|^{2+\frac{4}{N}} d x \\
& \leq \lambda_{k}^{2} E\left(u_{0}\right) \rightarrow 0, \text { as } k \rightarrow \infty .
\end{aligned}
$$

On the other hand, by (3.26) and the Gagliardo-Nirenberg inequality, one has $H\left(U_{k}\right) \geq 0$ and

$$
\lim _{k \rightarrow \infty} H\left(U_{k}\right)=0
$$

Combining (3.28) with (3.27), one has

$$
\lim _{k \rightarrow \infty}\left\|U_{k}\right\|_{L^{2+\frac{4}{N}}}^{2+\frac{4}{N}}=1+\frac{2}{N} .
$$

Applying Lemma 2.4 to the sequence $U_{k}\left(M=1, m^{2+4 / N}=1+\frac{2}{N}\right)$, one has that there exist $\left\{y_{k}\right\} \subset R^{N}$ and $U \in H^{1}$ such that

$$
U_{k}\left(x+y_{k}\right) \rightarrow U(x) \text { weakly in } H^{1} \text {, with }\|U\|_{L^{2}} \geq\|Q\|_{L^{2}} .
$$

That is,

$$
\lambda_{k}^{\frac{N}{2}} u\left(t_{k}, \lambda_{k}\left(x+y_{k}\right)\right) \rightarrow U(x) \text { weakly in } H^{1},
$$

which implies that for every $A>0$,

$$
\lim _{k \rightarrow \infty} \inf \int_{|x| \leq A} \lambda_{k}^{N}\left|u\left(t_{k}, \lambda_{k}\left(x+y_{k}\right)\right)\right|^{2} d x \geq \int_{|x| \leq A}|U|^{2} d x .
$$

Since the assumption $\frac{a\left(t_{k}\right)}{\lambda\left(t_{k}\right)} \rightarrow \infty$, one has

$$
\lim _{k \rightarrow \infty} \inf \sup _{y \in R^{N}} \int_{|x-y| \leq a\left(t_{k}\right)}\left|u\left(t_{k}, x\right)\right|^{2} d x \geq \int_{|x| \leq A}|U|^{2} d x .
$$

For every $A>0$, one has

$$
\lim _{k \rightarrow \infty} \inf \sup _{y \in R^{N}} \int_{|x-y| \leq a\left(t_{k}\right)}\left|u\left(t_{k}, x\right)\right|^{2} d x \geq \int|U|^{2} d x \geq \int Q^{2} d x .
$$

Therefore, since the sequence $\left\{t_{k}\right\}$ is arbitrary, one has

$$
\lim _{t \rightarrow T} \inf \sup _{y \in R^{N}} \int_{|x-y| \leq a(t)}|u(t, x)|^{2} d x \geq \int Q^{2} d x .
$$

On the other hand, for every $t \in\left[t_{0}, T\right)$, the function $y \mapsto \int_{|x-y| \leq a(t)}$ $|u(t, x)|^{2} d x$ is continuous and goes to 0 at infinity. It follows that

$$
\sup _{y \in R^{N}} \int_{|x-y| \leq a(t)}|u(t, x)|^{2} d x=\int_{|x-y(t)| \leq a(t)}|u(t, x)|^{2} d x,
$$

for some $y(t) \in R^{N}$. This completes the proof of Theorem 3.1. 


\section{Limiting profiles in $\Sigma$}

In this section, we obtain the limiting profiles of the Cauchy problem (1.1)(1.2) in the natural energy space $\Sigma$. Using the concentration properties obtained in Sect. 3 and the variational characterization of $Q(x)$, we obtain that if the initial data $u_{0} \in \Sigma$ with $\left\|u_{0}\right\|_{L^{2}}=\|Q\|_{L^{2}}$, then the blow-up solution remains close to $Q$ in $H^{1}\left(R^{N}\right)$ up to scaling and phase parameters, and also translation in the nonradial case. Moreover, we obtain the decomposition of blow-up solutions $u(t, x)$ with initial data $\|Q\|_{L^{2}}<\left\|u_{0}\right\|_{L^{2}}<\|Q\|_{L^{2}}+\alpha^{\prime}$.

Theorem 4.1. Let $u_{0} \in \Sigma$ and $\left\|u_{0}\right\|_{L^{2}}=\|Q\|_{L^{2}}$. Suppose that $t_{0}=\frac{\pi}{2 \omega}$ and the corresponding solution of the Cauchy problem (1.1)-(1.2) u(t,x) blows up in finite time $T \leq \frac{\pi}{\omega}$. Then there are functions $y(t) \in R^{N}, \gamma(t) \in R$ such that

$$
\lambda^{\frac{N}{2}}(t) u(t, \lambda(t)(x+y(t))) e^{i \gamma(t)} \rightarrow Q(x) \text { strongly in } \Sigma \quad \text { as } t \rightarrow T,
$$

where $\lambda(t)=\frac{\|\nabla Q\|_{L^{2}}}{\|\nabla u\|_{L^{2}}}$ and $Q(x)$ is the unique ground state solution of Eq. (1.5).

Proof. Applying Proposition 3.1, $\forall \varepsilon>0, \exists \delta>0$ s.t. when $|t-T|<\delta$, there are functions $y(t) \in R^{N}, \gamma(t) \in R$ such that

$$
\int\left|\lambda^{\frac{N}{2}}(t) u(t, \lambda(t)(x+y(t))) e^{i \gamma(t)}-Q(x)\right|^{2} d x<\frac{\varepsilon}{3|R|^{2}},
$$

where the positive constant $R$ is sufficiently large and chosen by later. and

By Theorem 3.2, one has that there exists an $y_{1} \in R^{N}$ such that $\left|y_{1}\right| \leq R_{0}$

$$
\begin{gathered}
\lim _{t \rightarrow T} y(t)=y_{1}, \\
|u(t, x)|^{2} \rightarrow\|Q\|_{L^{2}}^{2} \delta_{x=y_{1}}, \quad \text { as } \mathrm{t} \rightarrow \mathrm{T} .
\end{gathered}
$$

Now, for a sufficiently large $R>R_{0}>0$, one has

$$
\begin{aligned}
\int & |x|^{2}\left|\lambda^{\frac{N}{2}}(t) u(t, \lambda(t)(x+y(t))) e^{i \gamma(t)}-Q(x)\right|^{2} d x \\
= & \left\{\int_{|x| \leq R}+\int_{|x|>R}\right\}|x|^{2}\left|\lambda^{\frac{N}{2}}(t) u(t, \lambda(t)(x+y(t))) e^{i \gamma(t)}-Q(x)\right|^{2} d x \\
\leq & |R|^{2} \int\left|\lambda^{\frac{N}{2}}(t) u(t, \lambda(t)(x+y(t))) e^{i \gamma(t)}-Q(x)\right|^{2} d x \\
& +\int_{|x|>R}|x|^{2}\left|\lambda^{\frac{N}{2}}(t) u(t, \lambda(t)(x+y(t))) e^{i \gamma(t)}\right|^{2} d x \\
& +\int_{|x|>R}|x|^{2}|Q(x)|^{2} d x \\
= & I+I I+I I I .
\end{aligned}
$$

Applying (4.2), one has

$$
|I|<\frac{\varepsilon}{3} .
$$


Since $R>R_{0}>0,(4.2)$ and (4.3), one has

$$
\begin{aligned}
|I I| & \leq \int_{|x|>R}|x|^{2}\left|\lambda^{\frac{N}{2}}(t) u(t, \lambda(t)(x+y(t)))\right|^{2} d x \\
& \leq \int_{|z|>\lambda(t)(R+y(t))}\left|\frac{z}{\lambda(t)}-y(t)\right|^{2}|u(t, z)|^{2} d z \\
& \leq \int_{|z|>\lambda(t)\left(R+y_{1}\right)}\left|\frac{z}{\lambda(t)}-y(t)\right|^{2}\|Q(z)\|_{L^{2}}^{2} \delta_{z=x_{1}} d z \\
& \leq \frac{\varepsilon}{3} .
\end{aligned}
$$

Since $Q(x)$ is the positive and spherically symmetric solution with exponentially decay at infinity (see Lemma 2.2), one has

$$
|I I I|=\int_{|x|>R}|x|^{2}|Q(x)|^{2} d x<\frac{\varepsilon}{3},
$$

for sufficiently large $R>0$.

Therefore, by (4.5)-(4.8), one has

$$
\left.\left|\int\right| x\right|^{2}\left|\lambda^{\frac{N}{2}}(t) u(t, \lambda(t)(x+y(t))) e^{i \gamma(t)}-Q(x)\right|^{2} d x \mid<\varepsilon .
$$

Combining with Proposition 3.1, we have (4.1) holds in $\Sigma$. This completes the proof.

At the end of this section, using the variational characterization of Eq. (1.5) and scaling technique, we prove that if the initial data $u_{0} \in \Sigma$ close to the ground state $Q(x)$, then the blow-up solution $u(t, x)$ remains close to $Q(x)$ in $\Sigma$.

Theorem 4.2. Let $u_{0} \in \Sigma$ such that there exists a $\alpha_{1}>0$ such that the following property is true. For all $0<\alpha^{\prime} \leq \alpha_{1}$, there exists $\delta\left(\alpha^{\prime}\right)$ with $\delta\left(\alpha^{\prime}\right) \rightarrow 0$ as $\alpha^{\prime} \rightarrow 0$ such that for all $u \in H^{1}$,

$$
0<\alpha(u)=\alpha\left(u_{0}\right)=\int\left|u_{0}\right|^{2}-\int Q^{2}<\alpha^{\prime} .
$$

Further assuming $t_{0}=\frac{\pi}{2 \omega}$ and the corresponding solution of the Cauchy problem (1.1)-(1.2) $u(t, x)$ blows up in finite time $T \leq \frac{\pi}{\omega}$. Then there are functions $y(t) \in R^{N}$ and $\gamma(t) \in R$ such that

$$
\left\|\lambda^{\frac{N}{2}}(t) u(t, \lambda(t)(x+y(t))) e^{i \gamma(t)}-Q(x)\right\|_{\Sigma} \leq \delta\left(\alpha^{\prime}\right)
$$

where $\lambda(t)=\frac{\|\nabla Q\|_{L^{2}}}{\|\nabla u\|_{L^{2}}}$ and $Q(x)$ is the unique ground state solution of Eq. (1.5).

Proof. We prove this conclusion by contradiction. We show that for any sequence $t_{k} \rightarrow T$, there is a subsequence $t_{k_{j}}, y_{k_{j}}$ and $\gamma\left(t_{k_{j}}\right)$ such that

$$
\left\|\lambda^{\frac{N}{2}}\left(t_{k_{j}}\right) u\left(\lambda\left(t_{k_{j}}\right)\left(x+y\left(t_{k_{j}}\right)\right), t_{k_{j}}\right) e^{i \gamma\left(t_{k_{j}}\right)}-Q(x)\right\|_{\Sigma} \leq \delta\left(\alpha^{\prime}\right),
$$

where $Q(x)$ is the unique ground state solution of Eq. (1.5). If not, then (4.12) does not holds along some sequence $t_{k_{r}}$. But then we can find a subsequence 
of $t_{k_{r}}$ along with (4.12) holds, this is a contradiction. Since $t_{k}$ is an arbitrary sequence approaching $T$, and (4.11) follows.

Assume that for any $t_{n} \rightarrow T$, there is a subsequence $t_{n_{k}}$ such that $u_{n_{k}}:=$ $u\left(t_{n_{k}}, x\right) \in \Sigma$ such that

$$
\lim _{k \rightarrow \infty} \int\left|u_{n_{k}}\right|^{2} d x=\int Q^{2} d x \text { and } \lim _{k \rightarrow \infty}\left\|\nabla u_{n_{k}}\right\|_{L^{2}}^{2}=\infty
$$

Consider now $U_{n_{k}}=\lambda_{n_{k}}^{\frac{N}{2}} u_{n_{k}}\left(\lambda_{n_{k}} x\right)$, where $\lambda_{n_{k}}=\frac{\|\nabla Q\|_{L^{2}}}{\left\|\nabla u_{n_{k}}\right\|_{L^{2}}}$. We have the following properties for $U_{n_{k}}$

$$
\begin{gathered}
\left\|U_{n_{k}}\right\|_{L^{2}} \rightarrow\|Q\|_{L^{2}}, \\
\left\|\nabla U_{n_{k}}\right\|_{L^{2}}=1, \\
\lim _{k \rightarrow \infty} H\left(U_{n_{k}}\right) \leq \lim _{n \rightarrow \infty} \lambda_{n_{k}}^{2} E\left(u_{n_{k}}\right) \leq \lim _{n \rightarrow \infty} \lambda_{n_{k}}^{2} E\left(u_{0}\right)=0,
\end{gathered}
$$

by $(3.5)$.

On the other hand, $H\left(U_{n_{k}}\right) \geq\left[1-\left(\frac{\left\|U_{n_{k}}\right\|_{L^{2}}}{\|Q\|_{L^{2}}}\right)^{\frac{4}{N}}\right]\left\|\nabla U_{n_{k}}\right\|_{L^{2}}^{2}$ by Lemma 2.3, thus $\lim _{k \rightarrow \infty} H\left(U_{n_{k}}\right)=0$.

Now we take

$$
w_{n_{k}}=\frac{\|Q\|_{L^{2}}}{\left\|U_{n_{k}}\right\|_{L^{2}}} U_{n_{k}},
$$

we have $\left\|w_{n_{k}}\right\|_{L^{2}}=\|Q\|_{L^{2}},\left\|\nabla w_{n_{k}}\right\|_{L^{2}} \rightarrow 1$ and $H\left(w_{n_{k}}\right) \rightarrow 0$ as $k \rightarrow \infty$. Using the same procedure in Proposition 3.1 and Theorem 4.1, we have the conclusion, and this completes the proof.

\section{References}

[1] Brézis, H., Lieb, E.H.: A relation between pointwise convergence of functions and convergence of functionals. Proc. Am. Math. Soc. 88, 486-490 (1983)

[2] Cazenave, T.: Semilinear Schrödinger equations, Courant Lecture Notes in Mathematics, 10, NYU, CIMS, AMS (2003)

[3] Chen, J.Q., Guo, B.L.: Blow-up profile to the solutions of two-coupled Schrödinger equations. J. Math. Phys. 50, 023505 (2009)

[4] Damergi, I., Goubet, O.: Blow-up solutions to the nonlinear Schrödinger equation with oscillating nonlinearities. J. Math. Anal. Appl. 352, 336-344 (2009)

[5] Ginibre, J., Velo, G.: On a class of nonlinear Schrödinger equations. I. The Cauchy problem, general case. J. Funct. Anal. 32, 1-32 (1979)

[6] Glassey, R.T.: On the blowing up of solutions to the Cauchy problem for nonlinear Schrödinger equations. J. Math. Phys. 18, 1794-1797 (1977)

[7] Gerard, P.: Description du defaut de compacite de l'injection de Sobolev. ESAIM Control Optim. Calc. Var. 3, 213-233 (1998) 
[8] Hmidi, T., Keraani, S.: Blowup theory for the critical nonlinear Schrödinger equations revisited. Int. Math. Res. Not. 46, 2815-2828 (2005)

[9] Kwong, M.K.: Uniqueness of positive solutions of $\triangle u-u+u^{p}=0$ in $R^{n}$. Arch. Rational. Mech. Anal. 105, 243-266 (1989)

[10] Konotop, V., Pacciani, P.: On collapse in the nonlinear Schröinger equation with time dependent nonlinearity. Application to Bose-Einstein condensates, arXiv:cond-mat/0504493v1, 19 April 2005

[11] Kunze, M.: Infinitely many radial solutions of a variational problem related to dispersion-managed optic fibers. Proc. Am. Math. Soc. 131(7), 2181-2188 (2002)

[12] Montesinos, G.D., Pérez-Garcia, V.M.: Numerical studies of stabilized Townes solitons. Math. Comput. Simul. 69, 447-456 (2005)

[13] Merle, F., Tsutsumi, Y.: $L^{2}$ concentration of blow up solutions for the nonlinear Schrödinger equation with critical power nonlinearity. J. Diff. Equ. 84, 205-214 (1990)

[14] Merle, F.: Determination of blow-up solutions with minimal mass for nonlinear Schrödinger equations with critical power. Duke Math. J. 69(2), 427-454 (1993)

[15] Merle, F., Raphaël, P.: Blow-up dynamic and upper bound on the blow-up rate for critical nonlinear Schrödinger equation. Ann. Math. 16, 157-222 (2005)

[16] Merle, F., Raphaël, P.: On a sharp lower bound on the blow-up rate for the $L^{2}$-critical nonlinear Schrödinger equation. J. Am. Math. Soc. 19, 37-90 (2006)

[17] Merle, F., Raphaël, P.: Profiles and quantization of the blowup mass for critical nonlinear Schrödinger equation. Comm. Math. Phys. 253, 675-704 (2005)

[18] Ogawa, T., Tsutsumi, Y.: Blow-up of $H^{1}$ solution for the nonlinear Schrödinger equation. J. Diff. Equ. 92, 317-330 (1991)

[19] Strauss, W.A.: Existence of solitary waves in higher dimensions. Comm. Math. Phys. 55, 149-162 (1977)

[20] Sulem C., Sulem, P.L.: The nonlinear Schröinger Equation. In: Self-Focusing and Wave Collapse, Applied Mathematics Science, vol. 139. Springer-Verlag, New York (1999)

[21] Weinstein, M.I.: Nonlinear Schrödinger equations and sharp interpolation estimates. Comm. Math. Phys. 87, 567-576 (1983)

[22] Weinstein, M.I.: On the structure and formation of singularities in solutions to nonlinear dispersive evolution equations. Comm. Part. Diff. Equ. 11, 545-565 (1986)

[23] Zhang, J.: Sharp conditions of global existence for nonlinear Schrödinger and Klein-Gordon equations. Nonlinear Anal. 48, 191-207 (2002)

[24] Zhang, J.: Sharp threshold for blowup and global existence in nonlinear Schrödinger equation under a harmonic potential. Comm. Partial Diff. Equ. 30, 1429-1443 (2005) 
[25] Zhu, S.H., Zhang, J., Li, X.G.: Limiting profile of blow-up solutions for the Gross-Pitaevskii equation. Sci. Chin. Ser. A 52, 1017-1030 (2009)

[26] Zharnitsky, V., Grenier, E., Jones, C., Turitsyn, S.: Stabilizing effects of dispersion management. Phys. D 152-153 (2001), 794-817

Jian Zhang and Shihui Zhu

College of Mathematics and Software Science

Sichuan Normal University

Chengdu 610066

China

Shihui Zhu

College of Mathematics and Software Science

Sichuan Normal University

No. 5 Jing'an Road

Jinjiang District

Chengdu

Sichuan Province 610066

China

e-mail: zhu_shihui2008@163.com

and

College of Mathematics

Sichuan University

Chengdu 610064

China

Received: 17 September 2010.

Accepted: 6 June 2011. 